\title{
UPSCALING AND VALIDATION OF RTK-DIRECT GEOREFERENCED UAV-BASED RGB IMAGE DATA WITH PLANET IMAGERY USING POLYGON GRIDS FOR PASTURE MONITORING
}

\author{
G. Bareth ${ }^{1 *}$ and C. Hütt ${ }^{1}$ \\ ${ }^{1}$ GIS \& RS Group, Institute of Geography, University of Cologne, 50923 Cologne, Germany - \\ (g.bareth, christoph.huett)@uni-koeln.de
}

Commission III, WG III/10 (Agriculture and Natural Ecosystems Modelling and Monitoring)

KEY WORDS: Grassland, Biomass, Forage, Sward, Height, Quality, Vegetation Index, 3D

\begin{abstract}
:
The monitoring of managed grasslands with remote sensing methods is becoming more important for spatial decision support. Various remote sensing data acquisition techniques are applied for that purpose in different spatial resolutions ranging from UAVborne to satellite-based remote sensing. In the last decade, UAV-borne imaging and analysis techniques or in the focus of crop and grassland monitoring and provide very high spatial resolutions. In contrast, satellite data are only available in high to moderate spatial resolutions. In this contribution, we introduce direct georeferenced data acquisition with a Phantom 4 RTK for pasture monitoring and investigate the upscaling of the $\mathrm{cm}$ data to satellite resolutions using polygon grids.
\end{abstract}

\section{INTRODUCTION}

Proximal and remote sensing technologies play a vital role in precision agriculture (Mulla 2013). For managed grasslands, monitoring approaches face more difficulties due to higher spatio-temporal variations in floristic composition and canopy traits (Schellberg et al. 2008). However, multitemporal satellite remote sensing and UAV-based sensing approaches are suitable to monitor grasslands (Rango et al. 2009, Reinermann et al. 2020). While proximal sensing with manual or tractor-based devices does not provide spatial coverage, optical remote sensing in spatial resolutions below $10 \mathrm{~m}$ is often limited due to cloud cover. UAV-based monitoring seems to fit well for these requirements providing very high spatial resolutions whenever necessary in terms of phenology or management, almost on daily base. A limitation for UAV-based approaches to monitor grasslands is costs. Therefore, fully automated UAV data acquisition and analysis workflows are required. For the latter purpose of developing analysis approaches towards a fully automated workflow, only RTK-direct georeferenced image data has the potential to provide desired spatial accuracy to derive spectral and structural canopy traits. Therefore, the objectives of this study are (i) the accuracy evaluation of RTKdirect georeferenced RGB image data, (ii) the upscaling of such data using polygon grids, and (iii) evaluation against highresolution optical satellite data.

\section{METHOD}

The UAV campaign was carried out at the pasture field experiment "Forbioben". The grazing trial is conducted by the Institute of Grassland Science at the Georg-August-University Göttingen, Germany, and is described in detail by Tonn et al. (2019). In 2002, the field experiment was established. Overall objective is the investigation of patch-grazing patterns. The experiment consists of 9 single 1 ha large paddocks in three intensity repetitions.
For the UAV data acquisition, a DJI Phantom 4 RTK (P4RTK) was used on 05 June 2020. The data acquisition was obtained from $40 \mathrm{~m}$ and $45 \mathrm{~m}$ above ground resulting in a spatial resolutuion of $0.013 \mathrm{~m}$. The P4RTK is equipped with a 1" CMOS sensor capturing RGB images with 20 megapixels. Stereo photogrammetric analyses using Structure from Motion and Multiview Stereopsis (SfM/MVS) were done with Agisoft Metashape. The SfM/MVS analysis result, the Digital Orthophoto (DOP), was exported in a GIS. For the latter ESRI ArcGIS was used.

For sward growth analysis, the single RGB bands of the DOP were further analysed in ArcGIS. Using the raster calculator function, the vegetation index RGBVI which was introduced by Bareth et al. (2015) and by Bendig et al. (2015), was computed.

$$
R G B V I=\frac{G^{2}-B * R}{G^{2}+B * R}
$$

The final step of the UAV data processing was the computation of zonal statistics using a $10 \mathrm{~m}$ polygon grid which was generated in ArcGIS. In Fig.1, the generated DOP and the $10 \mathrm{~m}$ polygon grid are shown. Additionally, the layout of the grazing experiment can be seen in Fig.1. The nine 1 ha large paddocks are visualized in black bold outlines and the GCPs are colored in red.

Besides the UAV-derived RGB DOP, a high resolution multispectral satellite image was obtained for further analysis. A high-resolution satellite image was acquired by one of Planet's Scope CubeSats on 02 June 2020. The image has a spatial resolution of $\sim 3 \mathrm{~m}$ and consists of four spectral bands (Blue: 455-515 nm, Green: 500-590 nm, Red: 590-670 nm, NIR: $780-860 \mathrm{~nm}$ ). It was provided by Planet orthorectified and with an applied atmospheric correction (Planet Team 2017). From the Planet data, the vegetation indices RGBVI and NDVI were computed:

\footnotetext{
* Corresponding author
} 


$$
N D V I=\frac{N I R-R}{N I R+R}
$$

Bareth et al. (2016) introduced for upscaling UAV-derived data in ultra-high-spatial resolution the usage of continuous polygon grids and zonal statistics. Zonal statistics are an established GIS functionality which is used to compute descriptive statistics and/or histograms for certain selected zones. To our knowledge, the usage of zonal statistics for a continuous vector grid, the polygon grid, is not applied and provides interesting solutions for upscaling UAV data.. In this approach, every single vector cell serves as a unique zone. The vector grid size can be adjusted to the desired resolution very easily. So, both data sets, the UAV-derived RGBVI and the RGBVI/NDVI from the Planet image were upscaled by using a $10 \mathrm{~m}$ polygon grid. In Fig.1, the DOP and the $10 \mathrm{~m}$ polygon grid are shown.

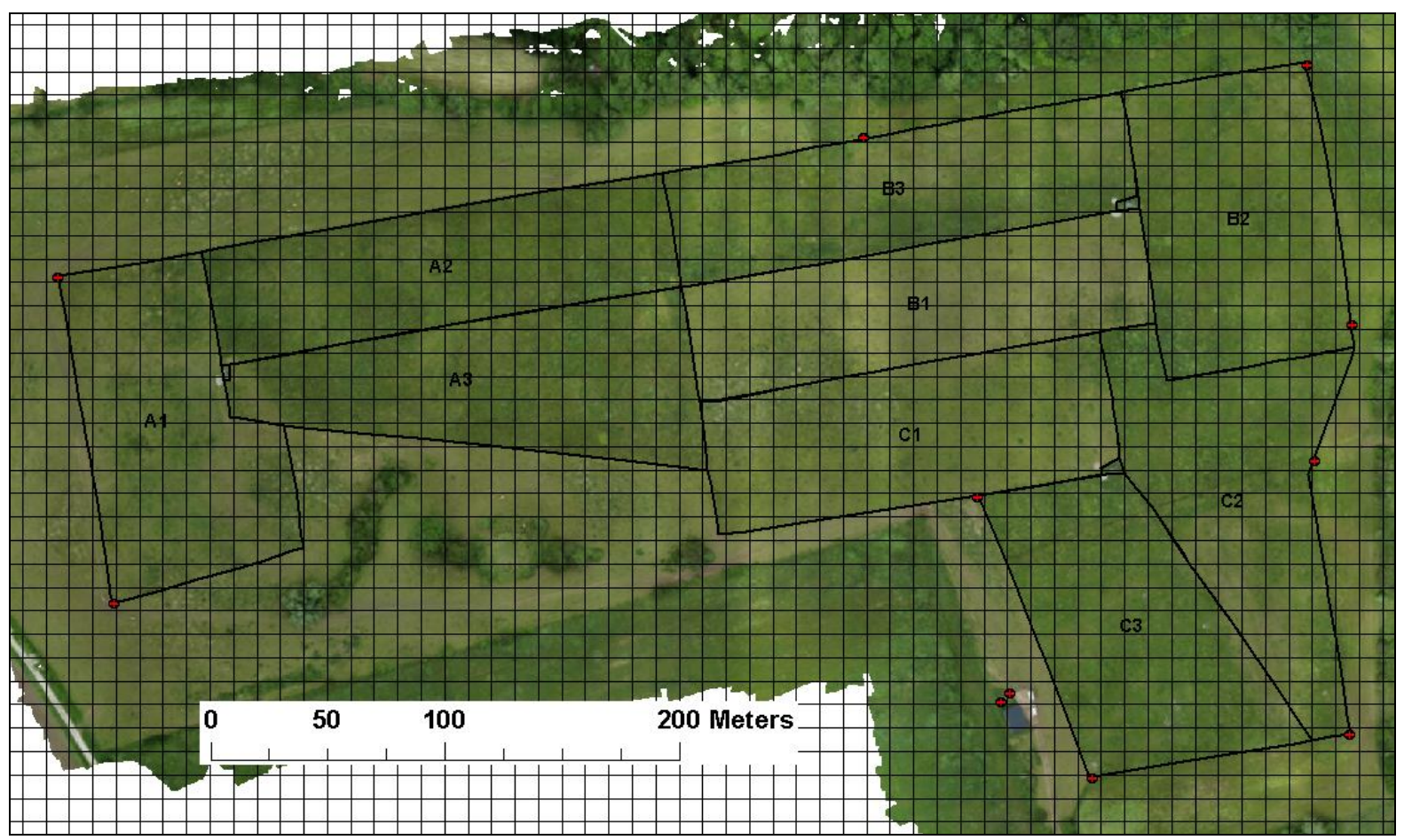

Figure 1. UAV-derived orthophoto of the grazing experiment "FORBIOBEN" and the $10 \mathrm{~m}$ polygon grid. The pasture experiment is conducted by the Institute of Grassland Science, University of Göttingen.

\section{RESULTS}

The first objective of this contribution is the accuracy evaluation of the RTK-direct georeferenced RGB image data. The spatial accuracy was evaluated using ten Ground Control Points (GCPs) directly located at the outlines of the paddocks and very close to the base station. (see Fig.1). The RMSE for $\mathrm{x}$ and $\mathrm{y}$ is $0.047 \mathrm{~m}$. The results are visualized in Fig. 2 for GCP1 and GCP3.

The RGBVI was computed on the $0.013 \mathrm{~cm}$ DOP and was upscaled via zonal statistics to the 10 m polygon grid. In Fig.3, the computed RGBVI in DOP resolution is shown while in Fig.4, the result of the upscaling of the UAV-derived RGBVI in $10 \mathrm{~m}$ is presented. It is very well recognizable that there is a significant spatial heterogeneity of the pasture and the overall spatial pattern of the two very different spatial resolutions is preserved. However, as expected is the upscaled pattern of the $10 \mathrm{~m}$ polygon grid significantly smoothed in comparison to the original resolution of the DOP.

In Fig.5 and Fig.6, a similar pattern can be observed for the Planet RGBVI and NDVI data. The Planet NDVI is also upscaled on the $10 \mathrm{~m}$ polygon grid using zonal statistics. Again it is clearly visible by comparing Fig.3, Fig.4, Fig.5, and Fig.6 that the spatial pattern of the UAV-derived RGBVI in DOP resolution of $0.013 \mathrm{~cm}$ and in $10 \mathrm{~m}$ is given in the Planet data as well.

Finally, the results of the Planet-NDVI are plotted cellwise against UAV-RGBVI $(\mathrm{n}=994)$. The two datasets correlate well, having a high $\mathrm{R}^{2}$ of 0.81 .

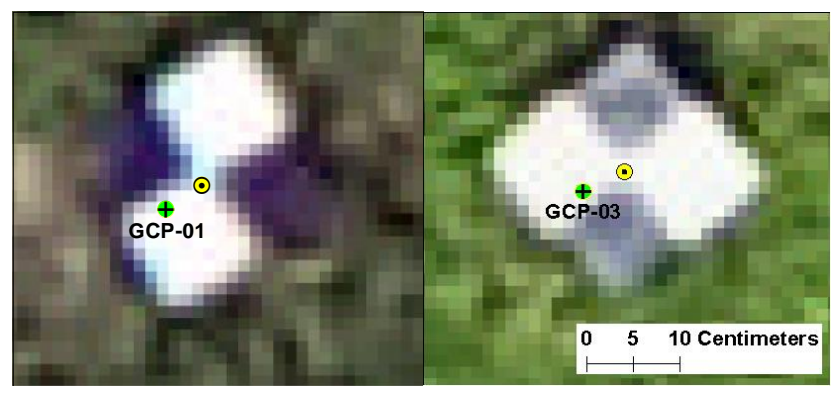

Figure 2. Spatial accuracy of the DOP (yellow) against two RTK-GPS measured markers (green) for June $5^{\text {th }}, 2020$ ( $\mathrm{x}, \mathrm{y}$-RMSE for all ten markers is $0.047 \mathrm{~m})$. 


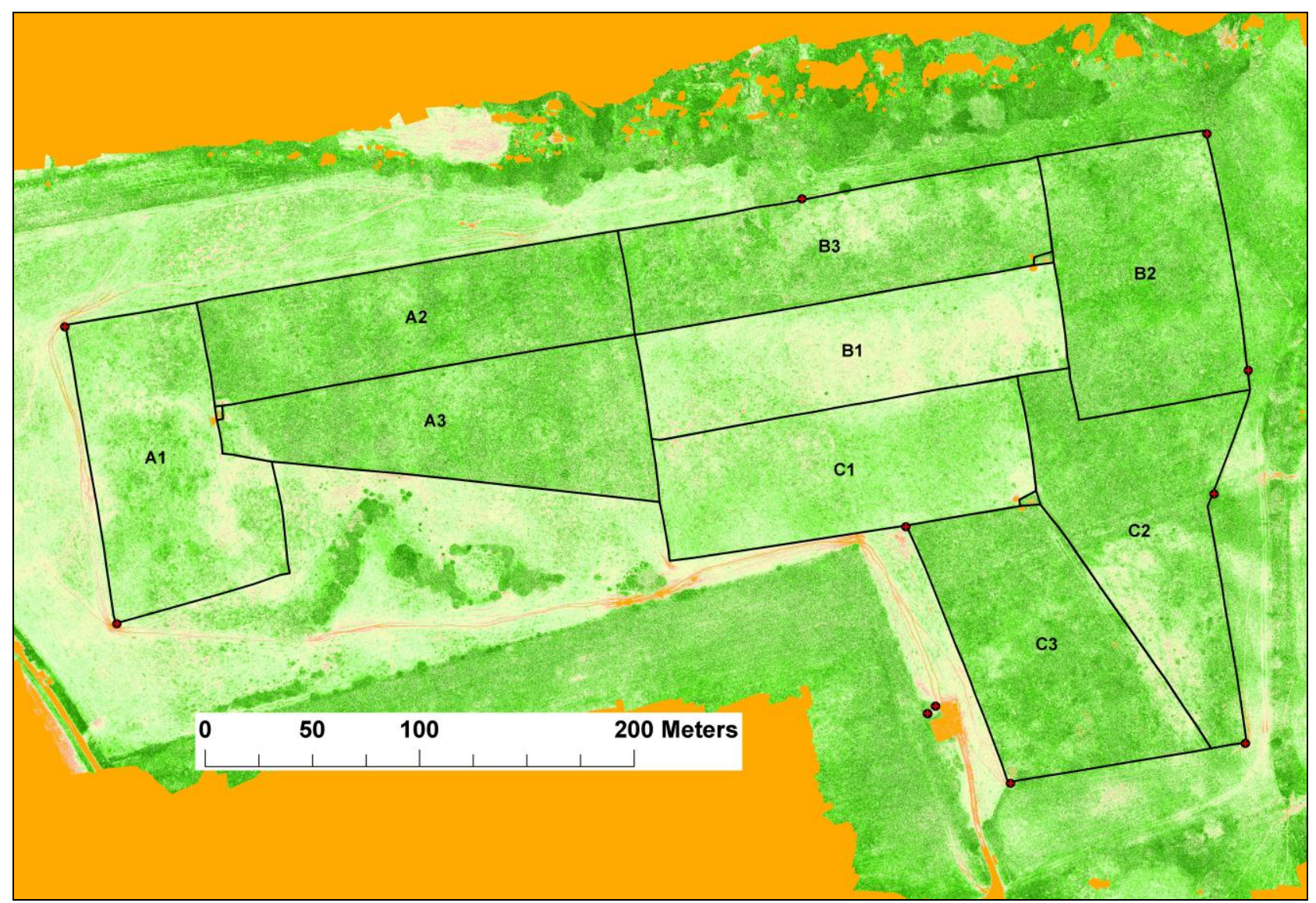

Figure 3. RGBVI analysis of the UAV-derived orthophoto in original spatial resolution of $0.013 \mathrm{~m}$ (05 June 2020).

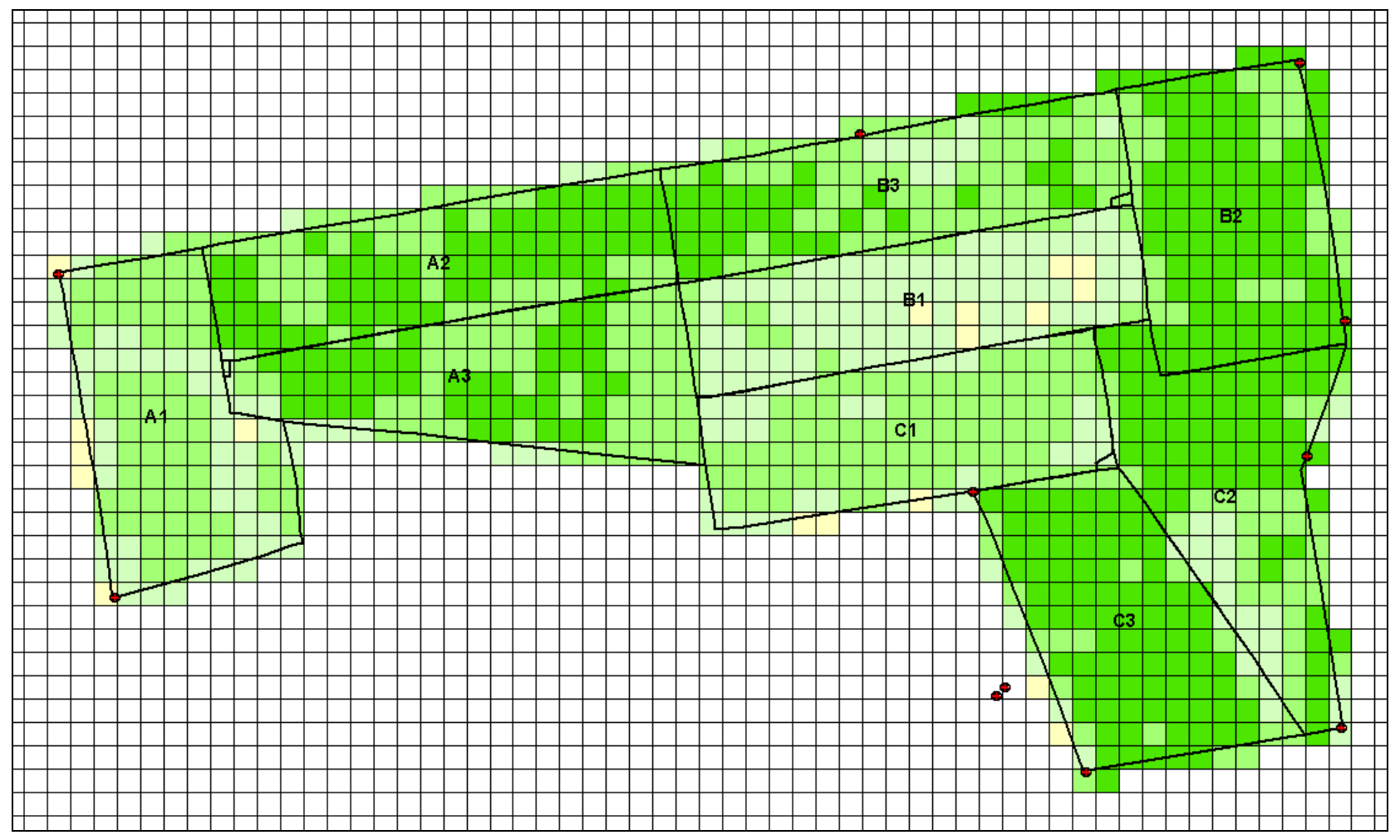

Figure 4. RGBVI analysis of the UAV-derived orthophoto for the $10 \mathrm{~m}$ polygon grid (05 June 2020). 


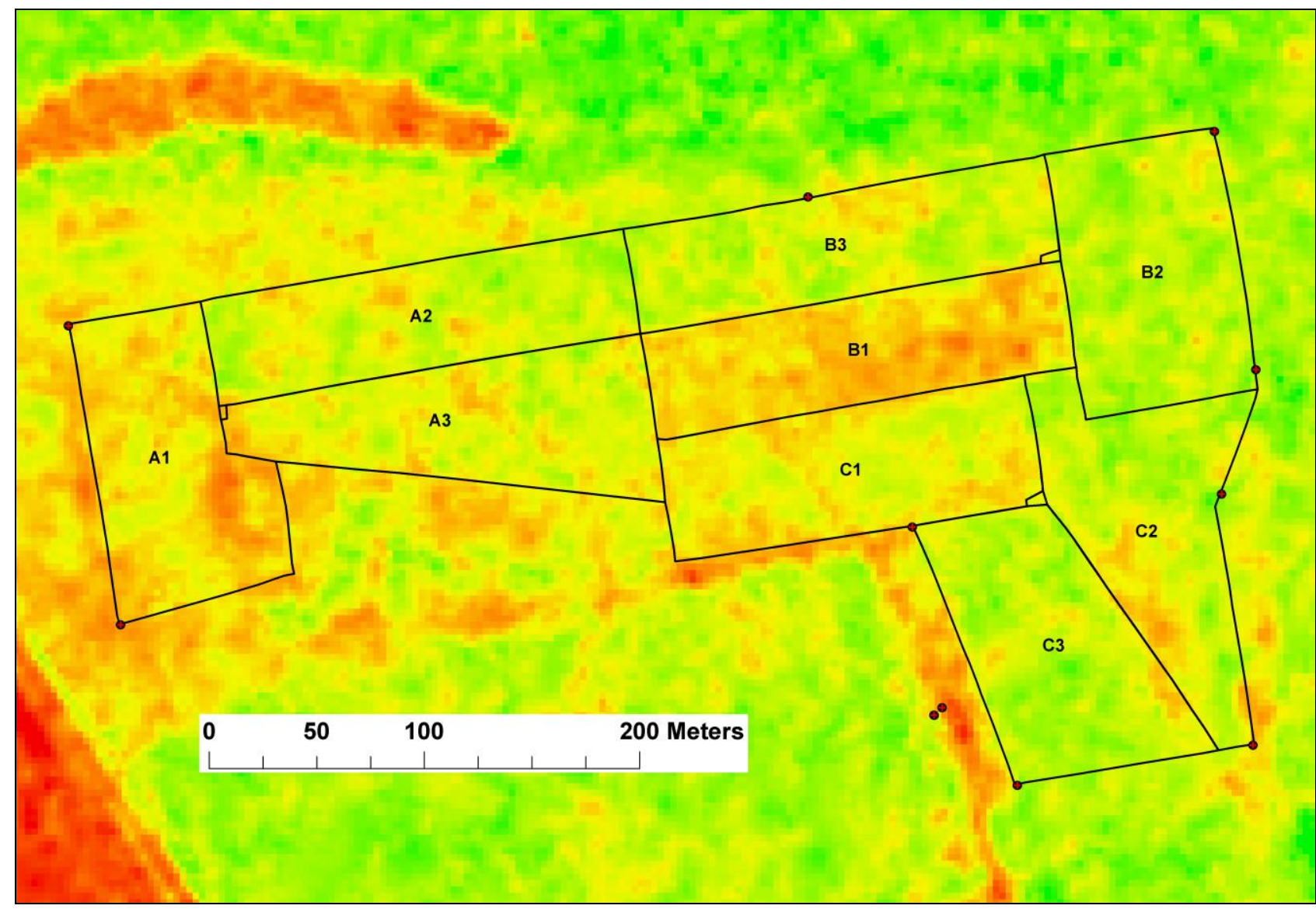

Figure 5. RGBVI analysis of the Planet image in original resolution of $\sim 3 \mathrm{~m}$ (02 June 2020).

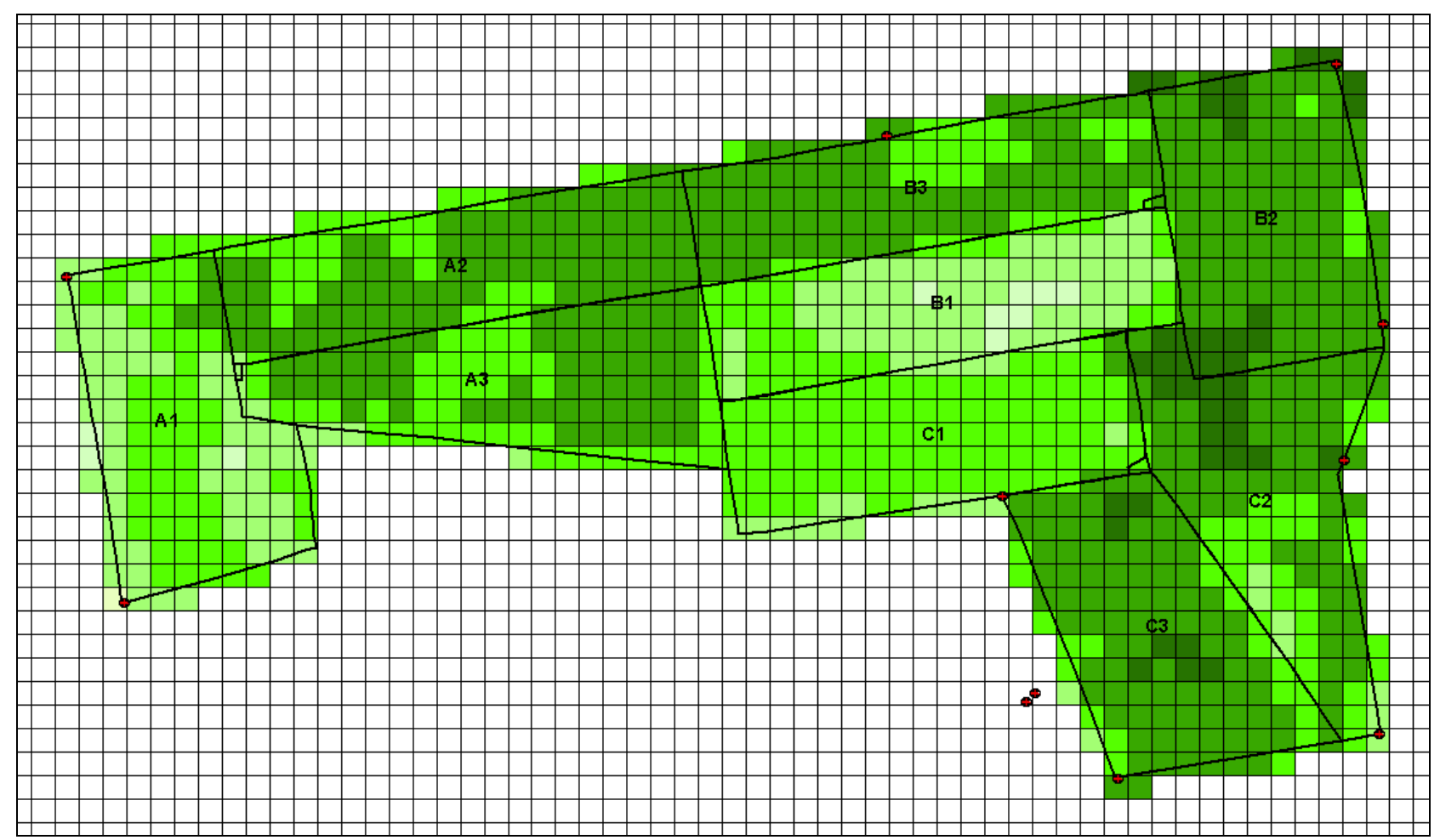

Figure 6. NDVI analysis of the Planet image for the $10 \mathrm{~m}$ polygon grid (02 June 2020). 


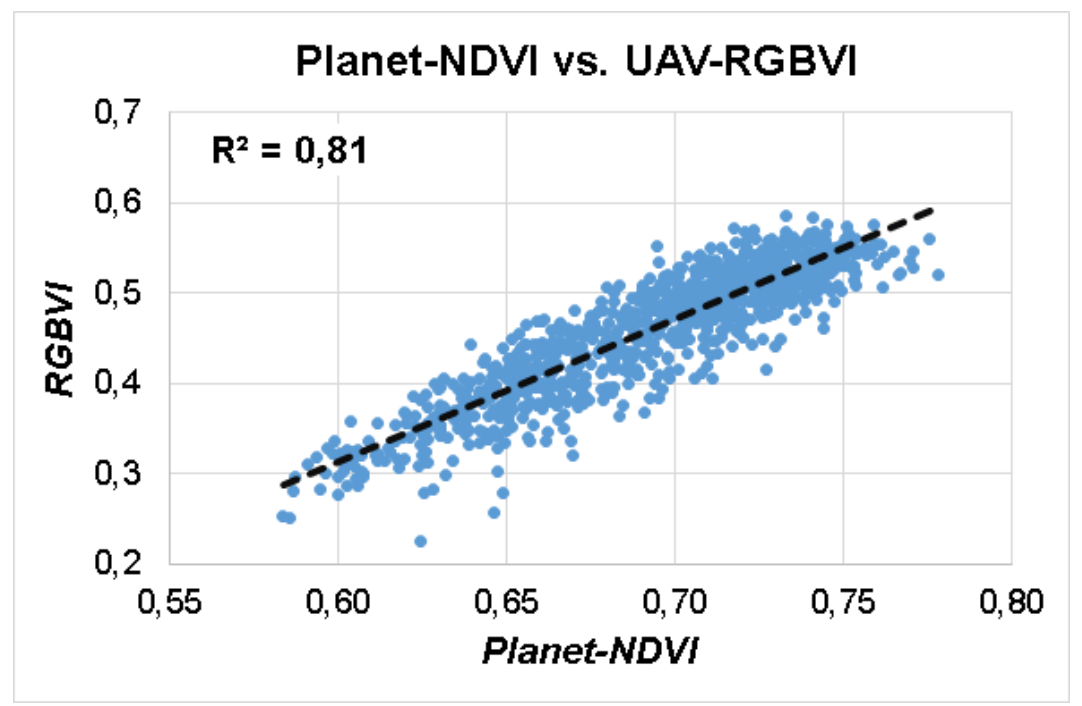

Figure 7. Planet NDVI vs. UAV RGBVI for the $10 \mathrm{~m}$ polygon grid $(\mathrm{n}=994)$.

\section{DISCUSSION AND CONCLUSIONS}

The three objectives of this study (i) the accuracy evaluation of RTK-direct georeferenced RGB image data, (ii) the upscaling of such data using polygon grids, and (iii) evaluation against highresolution optical satellite data are investigated. (i) The accuracy evaluation resulted in expected and reported results by the producer DJI (www.dji.com). A x,y-RMSE of approx. $5 \mathrm{~cm}$ for direct georeferencing using a base station is satisfying because the goal is to use the UAV derived data in spatial resolutions of 0.5 to $10 \mathrm{~m}$ (Bareth 2020; Bareth and Hütt 2020). (ii) The upscaling of the UAV data worked well and the visual spatial pattern seems to be preserved. For further evaluation, (iii) the UAV data was correlated against high resolution satellite data. Plotting the UAV-derived RGBVI against the Planet-derived NDVI in $10 \mathrm{~m}$ spatial resolution resulted in a high $\mathrm{R}^{2}$ of 0.81 . The latter is an interesting finding: the radiometrically uncalibrated UAV-RGB image seems to provide similar spatial patterns as the multispectral satellite remote sensing data. Further analysis should be conducted using different satellite data. However, further analysis should also include sward height analysis, which is a robust estimator of forage mass (Lussem et al. 2020) and would enable combined analysis of spectral and structural analysis workflows like the one introduced by Viljanen et al. (2018).

\section{ACKNOWLEDGEMENTS}

This study was conducted for the "GreenGrass" project (www.greengrass-project.de) which is funded by the Federal Ministry of Education and Research (BMBF) [Grant number 031B0734F]. The "FORBIOBEN" pasture experiment is conducted by the Institute of Grassland Science of the University of Göttingen.

\section{REFERENCES}

Bareth, G. (2021, accepted): Towards an informed grassland farming - Sensors, platforms and algorithms. 21st EGF Symposium 2021 Sensing - New Insights into Grassland Science and Practise.

Bareth, G. and Hütt, C. (2021, accepted): Using polygon grids to upscale ultra-high resolution UAV data for monitoring pastures. 21st EGF Symposium 2021 Sensing - New Insights into Grassland Science and Practice.

Bareth G., Bendig J., Tilly N., Hoffmeister D., Aasen H., and Bolten A. (2016) A comparison of UAV- and TLS-derived plant height for crop monitoring: using polygon grids for the analysis of crop surface models (CSMs). Photogramm Fernerkundung Geoinf 84, 85-94.

https://doi.org/10.1127/pfg/2016/0289

Bareth G, Bolten A, Hollberg J, Aasen H, Burkhart A, and Schellberg J (2015) Feasibility study of using non-calibrated UAV-based RGB imagery for grassland monitoring: case study at the Rengen Long-term Grassland Experiment (RGE), Germany. In: DGPF annual conference'15, Cologne, Germany, pp 55-62.

https://dgpf.de/src/tagung/jt2015/proceedings/papers/07_DGPF 2015_Bareth_et_al.pdf.

Bendig J, Yu K, Aasen H, Bolten A, Bennertz S, Broscheit J, Gnyp ML, and Bareth G (2015) Combining UAV-based plant height from crop surface models, visible, and near infrared vegetation indices for biomass monitoring in barley. Int J Appl Earth Obs Geoinform 39:79-87.

https://doi.org/10.1016/j.jag.2015.02.012

Lussem U., Schellberg J., and Bareth G. (2020) Monitoring forage mass with low-cost UAV data: case study at the Rengen grassland experiment. PFG - J Photogramm Remote Sens Geoinf Sci. 88, 407-422.

https://doi.org/10.1007/s41064-020-00117-w 
Mulla, DJ (2013) Twenty five years of remote sensing in precision agriculture: Key advances and remaining knowledge gaps. Biosystems Engineering 114 (4), 358-371.

https://doi.org/10.1016/j.biosystemseng.2012.08.009

Planet Team (2017): Planet Application Program Interface: In Space for Life on Earth. San Francisco, CA. https://api.planet.com.

Rango A., Laliberte A., Herrick J.E., Winters C., Havstad K., Steele C., and Browning D. (2009) Unmanned aerial vehiclebased remote sensing for rangeland assessment, monitoring, and management. J Appl Remote Sens. 3, 033542.

https://doi.org/10.1117/1.3216822

Reinermann S, Asam S, and Kuenzer C. (2020) Remote Sensing of Grassland Production and Management-A Review. Remote Sensing 12(12), 1949. https://doi.org/10.3390/rs12121949

Schellberg, J., Hill, M.J., Gerhards, R., Rothmund, M., and Braun, M. (2008): Precision agriculture on grassland: Applications, perspectives and constraints. Eurp. J. Agronomy 29 (2-3), 59-71. https://doi.org/10.1016/j.eja.2008.05.005

Tonn B., Raab C., and Isselstein J. (2019) Sward patterns created by patch grazing are stable over more than a decade. Grass and Forage Science 74, 104-114.

https://doi.org/10.1111/gfs.12389

Viljanen N, Honkavaara E, Näsi R, Hakala T, Niemeläinen O, and Kaivosoja J (2018) A novel machine learning method for estimating biomass of grass swards using a photogrammetric canopy height model, images and vegetation indices captured by a drone. Agriculture 8(5):70.

https://doi.org/10.3390/agriculture8050070 